ISSN 1980-5098@@ (@) DOI: http://dx.doi.org/10.5902/1980509831589

\title{
ESTRUTURA POPULACIONAL E DISTRIBUIÇÃO ESPACIAL DE Theobroma speciosum Willd. Ex Spreng NO NORTE DO ESTADO DE MATO GROSSO
}

\author{
POPULATIONAL STRUCTURE AND SPATIAL DISTRIBUTION OF Theobroma speciosum \\ Willd. Ex Spreng IN NORTHERN OF MATO GROSSO STATE
}

\author{
Tatiane Lemos Varella ${ }^{1}$ Ana Aparecida Bandini Rossi ${ }^{2}$ Maicon Douglas Arenas de Souza ${ }^{3}$ \\ Greiciele Farias da Silveira ${ }^{4}$ Jakeline Santos Cochev ${ }^{5}$ José Julio de Toledo ${ }^{6}$ Carolina Joana da Silva ${ }^{7}$
}

\section{RESUMO}

Entender os padrões de estrutura e distribuição espacial das espécies de árvores é fundamental para prever a variação espacial de processos sucessionais, genéticos e ecológicos de um ecossistema florestal. Neste estudo foram analisadas a estrutura populacional e a distribuição espacial de Theobroma speciosum (cacauí) em três fragmentos florestais urbanos (Parques C/E com 6,72 ha amostrados, J com 3,92 ha e Zoobotânico com 15,84 ha) em Alta Floresta e no Parque Nacional do Juruena - PNJu (com 3,2 ha amostrados) no norte do estado de Mato Grosso. Foram amostrados 323 indivíduos de Theobroma speciosum, sendo 50 no fragmento do Parque C/E (7,44 ind. ha $\left.{ }^{-1}\right), 32$ no Parque J (8,16 ind.ha $\left.{ }^{-1}\right), 76$ no Parque Zoobotânico (4,79 ind. ha $\left.{ }^{-1}\right)$ e 165 no PNJu (51,56 ind. ha $\left.{ }^{-1}\right)$. A menor densidade de indivíduos encontrada nos parques urbanos pode estar relacionada com o processo de fragmentação, visto que não houve uma relação positiva da densidade e do número de indivíduos com a área amostral. Além disso, a maior densidade foi encontrada no $\mathrm{PNJu}$, que é uma unidade de conservação de floresta contínua e com nenhuma intervenção antrópica recente. Os fragmentos florestais urbanos (com exceção do Parque Zoobotânico) apresentaram uma menor quantidade de indivíduos nas classes inferiores de diâmetro $(0-5 \mathrm{~cm})$ e altura $(0-5 \mathrm{~m})$ com relação à estrutura encontrada no PNJu. Essa diferença provavelmente deve-se à menor área dos Parques $\mathrm{C} / \mathrm{E} \mathrm{e}$ $\mathrm{J}$ e consequentemente a um efeito de borda mais intenso que afetou a regeneração florestal. O padrão de distribuição dos indivíduos em todas as populações foi agregado. Esses resultados indicam que a espécie é susceptível à fragmentação provavelmente devido ao isolamento, pois apresenta autoincompatibilidade e uma dispersão zoocórica limitada.

Palavras-chave: cacauí; Amazônia; ecologia florestal.

1 Bióloga, MSc., Universidade do Estado de Mato Grosso, Rua dos Limoeiros, 884, Jardim Celeste, CEP 78556000, Sinop (MT), Brasil. tatvarella@gmail.com

2 Bióloga, Dra., Professora dos Programas de Pós-Graduação PPGBioAgro, Universidade do Estado de Mato Grosso, Rod. MT 208, KM 143, s/n, Jardim Tropical, CEP 78580-000, Alta Floresta (MT), Brasil. anabanrossi@unemat.br

3 Biólogo, MSc., Doutorando em Ciências Biológicas, Universidade Federal do Rio de Janeiro, Quinta da Boa Vista, s/n, São Cristóvão, CEP 20940-040, Rio de Janeiro (RJ), Brasil. m.arenasbot@gmail.com

4 Bióloga, MSc., Professora da Universidade do Estado de Mato Grosso, Rua São Luis, 147, Boa Nova 2, CEP 78580-000, Alta Floresta (MT), Brasil. greicielefarias@hotmail.com

5 Licenciada em Geografia, MSc., Doutoranda em Biodiversidade e Biotecnologia da Amazônia Legal, Universidade do Estado de Mato Grosso, Rod. MT 208, KM 143, s/n, Jardim Tropical, CEP 78580-000, Alta Floresta (MT), Brasil. jackcochev@gmail.com

6 Biólogo, Dr., Professor doPrograma de Pós-Graduação em Biodiversidade Tropical, Universidade Federal do Amapá, Rod. Juscelino Kubitschek, Km 02, Jardim Marco Zero, CEP 68902-280, Macapá (AP), Brasil. jjuliotoledo@gmail.com

7 Bióloga, Dra., Professora do Programa de Doutorado em Biodiversidade e Biotecnologia da Amzonia Legal, Universidade do Estado de Mato Grosso, Cidade Universitária, s/n, Santos Dumont CEP 78200-000, Cáceres (MT),Brasil.ecopanta@terra.com.br 


\begin{abstract}
Understanding the structure and spatial distribution of tree species is necessary to predict the spatial variation of successional, genetic and ecological processes of a forest ecosystem. The present study assessed the population structure and the spatial distribution of Theobroma speciosum (cacauí) in three urban forest fragments located at Alta Floresta (C/E Park with sampled area of 6.72 ha, J Park, with 3.92 ha and Zoobotanical Park with 15.84 ha of sampled area) and at Juruena National Park -PNJu (3.2 ha) in the North of Mato Grosso State. Three hundred and twenty-three individuals of Theobroma speciosum were sampled, 50 in the C/E Park (7.44 ind. ha $\left.{ }^{-1}\right), 32$ in J Park (8.16 ind. ha $\left.{ }^{-1}\right), 76$ in the Zoo-botanical Park (4.79 ind. ha ${ }^{-1}$ ) and 165 in PNJu (51.56 ind. ha ${ }^{-1}$ ). The lower density of individuals found in the urban parks may be associated with the fragmentation process, since there were no positive relationships of density and the number of individuals with the sampled area. Besides, the highest density was found at PNJu, which is a continuous forest in a conservation unit subjected to no recent human intervention. The urban forest fragments (except for the Zoo-botanical Park) have presented the smallest amount of individuals in the inferior diameter $(0-5 \mathrm{~cm})$ and the lowest height classes $(0-5 \mathrm{~m})$ when they were compared to the structure found at PNJu. Such difference must regard the smaller area of the $\mathrm{C} / \mathrm{E}$ and $\mathrm{J}$ Parks and the consequent more intense edge effect that have affected forest regeneration. The distribution pattern of individuals in all populations was clumped. These results show that the species is susceptible to fragmentation due to isolation, since it presents self-incompatibility and limited zoochoric dispersion.
\end{abstract}

Keywords: cacauí; Amazon; forest ecology.

\title{
INTRODUÇÃO
}

O cacauí (Theobroma speciosum Willd. ex Spreng) é uma espécie frutífera nativa da floresta Amazônica (DUCKE, 1953), desenvolve-se preferencialmente em mata de terra firme não inundável (CAVALCANTE, 1991). As comunidades humanas nativas da Amazônia consomem os frutos in natura, além de utilizá-los na produção de sucos, geleias e sorvetes (SADDI, 1977; FERRÃO, 2001). Estudos demonstram que a espécie é promissora para a produção de biodiesel (MIRANDA et al., 2004). Os ácidos graxos encontrados nas suas sementes possuem composição muito similar à encontrada na manteiga de cacau (GILABERT-ESCRIVÁ et al., 2002). Essas características indicam um alto potencial para utilização na indústria de alimentos e biocombustíveis. Entretanto, a espécie é dispersa na natureza e pouco cultivada (CAVALCANTE, 1991), sendo premente a necessidade de melhor entendimento da estrutura populacional nativa para prover subsídio a estratégias de manejo e propagação visando ao uso desse recurso da biodiversidade amazônica.

Em decorrência da mudança do uso da terra, a espécie vem sofrendo o efeito da fragmentação do seu habitat, na maioria das vezes, devido à substituição de parte da floresta por pastagem (OLIVEIRA et al., 2003; CARVALHO et al., 2005) trazendo como consequência a formação de mosaicos de vegetação remanescente (CARVALHO et al., 2010). A fragmentação causa redução do tamanho das populações naturais (ESTOPA et al., 2006), alterando o funcionamento e a organização dos ecossistemas florestais. Estas alterações causam a redução do fluxo gênico entre populações, afetando diretamente no padrão de distribuição espacial das espécies (LAURANCE; VASCONCELLOS, 2009). As alterações de distribuição das espécies na paisagem podem influenciar na dinâmica das populações e na composição das comunidades, tendo consequências negativas sobre processos ecossistêmicos, como ciclagem e manutenção dos nutrientes e do carbono (LAURANCE et al., 2004; LAURANCE; VASCONCELOS, 2009).

Em uma população de árvores, os indivíduos jovens são considerados preditores da estrutura da população futura (FELFILI et al., 2001). Logo, para que uma espécie arbórea se mantenha em uma comunidade florestal, espera-se encontrar grande representatividade dos indivíduos jovens. Pequena quantidade de indivíduos nas classes diamétricas menores pode ser consequência de distúrbios causados por fogo e/ou animais (MULLER-LANDAU et al., 2006). Por outro lado, uma distribuição com grande quantidade de indivíduos menores pode indicar que a floresta está em fase de regeneração devido a distúrbios que afetaram principalmente as árvores grandes, como secas (NEPSTAD et al., 2007), tempestades 
(TOLEDO et al., 2012) e fragmentação (NASCIMENTO; LAURANCE, 2004; LAURANCE et al., 2011). Conhecer a estrutura de tamanho de uma população arbórea é passo primordial para prever a permanência da espécie na floresta, tendo em vista que o deficit de indivíduos pequenos pode indicar baixa sobrevivência e recrutamento, ao passo que a ausência de indivíduos grandes e reprodutivamente viáveis indica uma deficiência na produção de propágulos.

Apesar da importância econômica e ecológica do gênero Theobroma, ainda existem poucos estudos com as espécies silvestres. Neste contexto, o presente estudo tem como objetivo comparar a estrutura populacional e a distribuição espacial de Theobroma speciosum (cacauí) em fragmentos florestais urbanos e em uma área de floresta contínua no Norte de Mato Grosso.

\section{MATERIAL E MÉTODOS}

\section{Área de estudo}

O estudo foi conduzido na área urbana do município de Alta Floresta, localizado no Norte do estado de Mato Grosso (Figura 1A). O clima é equatorial continental úmido com estação seca, temperatura anual entre $19,6^{\circ} \mathrm{C}$ a $32,4^{\circ} \mathrm{C}$ e precipitações de maio a setembro entre 250 e $300 \mathrm{~mm}$ e de outubro a abril entre 900 e $1000 \mathrm{~mm}$ (TARIFA, 2011). O município está a uma altitude de 300 a $400 \mathrm{~m}$ acima do nível do mar. Os solos predominantes são o Latossolo Vermelho-Amarelo e os Gleissolos. O relevo é formado pelo Planalto Apiacás-Sucurundi e a Depressão Interplanáltica Amazônia Meridional (ROSS, 2009).

A vegetação do município é constituída por Florestas Ombrófilas Abertas e Densas, Florestas Estacionais e Formações Secundárias (BRASIL, 2007). De acordo com Arenas et al. (2015), os fragmentos vegetais que se encontram na área urbana estão isolados e agrupados e não há formação de corredores verdes.

Foram selecionados três parques municipais que constituem fragmentos florestais urbanos no município de Alta Floresta - MT: Parque C/E (W56 5’24” e S952'48”) que compreende uma área de 9,19 ha, Parque J (W56 4'48” e S9 53'24") com uma área de 7,38 ha e o Parque Zoobotânico (W56 4'12" e S9 $\left.9^{\circ} 1^{\prime} 36^{\prime \prime}\right)$ que possui uma área de 17,6 ha (Figura 1B). Os três fragmentos estão localizados no perímetro urbano e sofreram influência antrópica, apresentando muitas clareiras, residências adjacentes, plantas exóticas, grande número de árvores caídas e visitações frequentes. As três áreas sofreram no passado (década de 70 e 80) exploração florestal, porém, na década de 90 foram transformadas em unidades de conservação (Parques Municipais), a partir de então permanecem sem exploração.

Foram utilizados também, os dados de um levantamento realizado em 2013 no Parque Nacional do Juruena (DARDENGO, 2014) (Figura 1C). O Parque Nacional do Juruena (PNJu) é uma unidade de conservação de proteção integral, administrada pelo Instituto Chico Mendes de Biodiversidade (ICMBio). Possui uma área de 1.958.203,56 ha, sendo que 60\% da área total do parque localizam-se no estado de Mato Grosso, distribuído pelos municípios de Apiacás, Cotriguaçu e Nova Bandeirantes. O restante da área está localizado no estado do Amazonas, distribuído pelos municípios de Apuí e Maués (PARQUE NACIONAL DO JURUENA, 2013). A área do PNJu avaliada neste estudo localiza-se no município de Apiacás, MT (W57\%48'17" e S95' 9") (Figura 1C). 


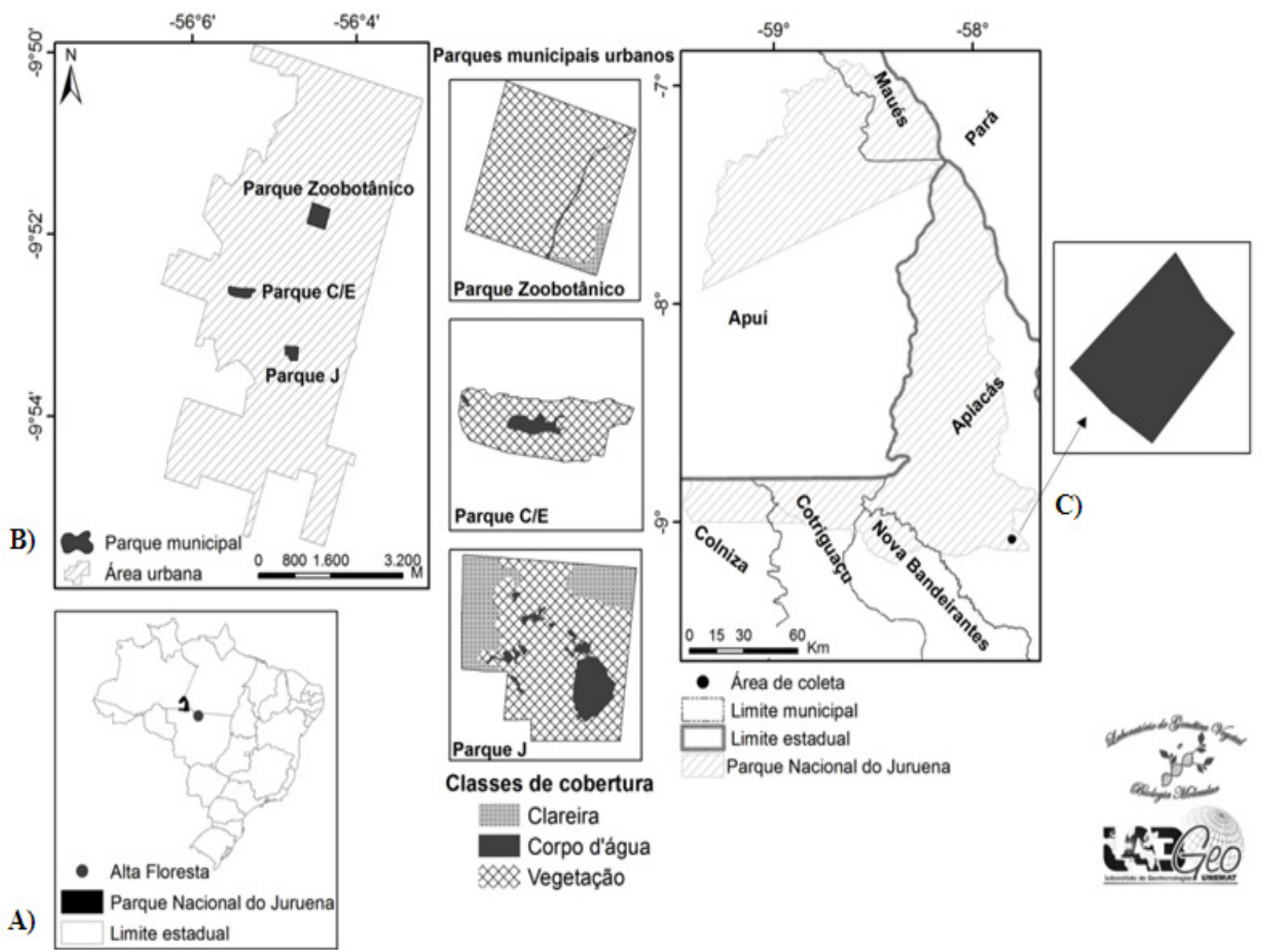

FIGURA 1: Localização geográfica da área de estudo. A) Localização do município de Alta Floresta - MT e do Parque Nacional do Juruena (PNJu). B) Localização dos três fragmentos urbanos e das parcelas estudadas no municipo de Alta Floresta - MT. C) Localização do PNJu e da parcela implantada. Fonte: Dardengo (2014).

FIGURE 1: Geographical location of the study area. A) Location of the municipality of Alta Floresta- MT and Juruena National Park (PNJu). B) Location of the three urban fragments and the studied plots in the municipality of Alta Floresta-MT. C) Location of the PNJu and the studied plot in MT state. Source: Dardengo (2014).

\section{Amostragem}

No Parque C/E foi implantada uma parcela de forma aleatória com dimensão de $420 \mathrm{~m} \times 160 \mathrm{~m}$ (6,72 ha) e dividida em 84 subparcelas de $20 \mathrm{~m} \times 40 \mathrm{~m}$. No Parque J foi instalada uma parcela de $280 \mathrm{~m}$ $\times 140 \mathrm{~m}(3,92 \mathrm{ha})$, dividida em 49 subparcelas $(20 \times 40)$, e no Parque Zoobotânico uma parcela de $440 \times$ $360 \mathrm{~m}$ (15,84 ha) dividida em 198 subparcelas $(20 \times 40)$.

No PNJu implantou-se uma parcela de $200 \mathrm{~m} \times 160 \mathrm{~m}$ (3,2 ha) dentro dos módulos do Programa de Pesquisa em Biodiversidade (PPBio), em que foram distribuídas 40 subparcelas de $20 \mathrm{~m} \times 40 \mathrm{~m}\left(800 \mathrm{~m}^{2}\right)$ (DARDENGO, 2014).

Dentro das parcelas foi mensurado o diâmetro de todos os indivíduos arbóreos vivos que apresentaram CAP (circunferência a altura do peito) $>1 \mathrm{~cm}$, sendo todos georreferenciados com auxílio do GPS Garmin Etrex ${ }^{\circledR}$, obtendo-se erros horizontais inferiores a $7 \mathrm{~m}$. Para os indivíduos que apresentaram ramificação, foi anotado apenas o maior valor de CAP. A altura foi estimada visualmente, tendo como base a altura de uma pessoa.

Os dados para testar a existência de diferenças entre áreas amostradas com relação às distribuições 
de diâmentro e altura dos indivíduos foram analisados através do teste de Kolmogorov-Smirnov em nível de significância de $5 \%$ de probabilidade (ZAR, 1966). As análises foram realizadas utilizando o software R (R DEVELOPMENT CORE TEAM, 2005).

O padrão de distribuição espacial foi estimado através do índice de Morisita (BROWER; ZAR, 1977):

$$
I_{d}=\frac{n\left[\sum\left(x_{i}^{2}\right)-\sum x_{i}\right]}{\sum\left(x_{i}\right)^{2}-\sum x_{i}}
$$

Em que $I_{d}$ é o índice de Morisita; $x_{i}$ é o número de indivíduos na subparcela $i$; e $n$ é número total de subparcelas amostradas.

A significância dos valores $I_{d}$ foi obtida mediante o teste do qui-quadrado $\left(\chi^{2}\right)$ em um nível de 0,05 . Valores de $I_{d}$ menores que 1 indicam uma distribuição regular, ao passo que valores iguais a 1 , ou que não diferem estatisticamente de 1 , indicam distribuição aleatória e valores maiores do que 1 , uma distribuição agregada (KREBS, 1989).

\section{RESULTADOS E DISCUSSÃO}

Foram amostrados 323 indivíduos de Theobroma speciosum nas quatro reservas, sendo 50 no fragmento do Parque C/E (7,44 ind. ha $\left.{ }^{-1}\right), 32$ no Parque J $\left(8,16\right.$ ind.ha $\left.a^{-1}\right), 76$ no Parque Zoobotânico (4,79 ind. ha $\left.{ }^{-1}\right)$ e 165 no PNJu $\left(51,56\right.$ ind. ha- $\left.{ }^{-1}\right)$. Tanto o número de indivíduos $\left(\chi^{2}=129,3, d f=3, P<0,001\right)$ quanto a densidade $\left(\chi^{2}=83,9, d f=3, P<0,001\right)$ diferiram entre reservas. Contudo, a relação entre o número de indivíduos e a área foi muito baixa (Correlação de Pearson usando dados logaritmizados: $r=-0,1 ; t=-0,15$, $d f=2, P=0,89)$, e embora não significativa $(t=-1,61, d f=2, P=0,24)$, a correlação entre a densidade de indivíduos e a área foi alta e negativa $(r=-0,75)$ indicando que outros fatores como histórico de uso e conservação podem ser determinantes da estrutura da população remanescente da espécie.

As parcelas dos parques urbanos (C/E, J e Zoobotânico) apresentaram menor densidade de indivíduos quando comparadas com a parcela do $\mathrm{PNJu}$, o que pode estar correlacionado com a fragmentação atual dos parques urbanos. Contudo, o PNJu é uma unidade de conservação permanente com floresta contínua, mantendo uma população de Theobroma speciosum livre de influência antrópica recente. Segundo Paglia, Fernandez e Marco (2006), fragmentos florestais apresentam menor diversidade de habitat do que florestas contínuas, o que pode alterar a estrutura da população (VASCONCELOS et al., 2006).

Densidades um pouco maiores que a encontrada nos parques urbanos deste estudo foram reportadas por Campbell et al. (1986), nas proximidades do Rio Xingú (Pará). Foi estimada uma densidade de 10,67 ind. ha ${ }^{-1}$ amostrando 48 indivíduos de Theobroma speciosum distribuídos em 4,5 ha. Entretanto, para outra espécie do gênero (Theobroma subincanum), Souza et al. (2011) amostraram 37 indivíduos em uma área de 9 ha, apresentando uma densidade de 4,1 ind. ha ${ }^{-1}$.

A distribuição diamétrica e de altura (Figuras 2 e 3 ) dos indivíduos de Theobroma speciosum nos parques Zoobotânico e PNJu tendeu a seguir uma função exponencial negativa "J invertido", que, segundo Machado et al. (2010), é característico da maioria das florestas nativas. Ribeiro, Fonseca e Silva (2001) relatam que isso ocorre no momento em que as populações de plantas apresentam uma predominância de indivíduos menores, decaindo exponencialmente à medida que se tornam maiores, indicando um balanço positivo entre recrutamento e mortalidade, sendo característico de populações autorregenerativas. Os indivíduos menores substituem os indivíduos adultos na população, permitindo a continuidade da sucessão ecológica nesses ambientes (SILVA JÚNIOR, 2004; CARVALHO; NASCIMENTO, 2009). 

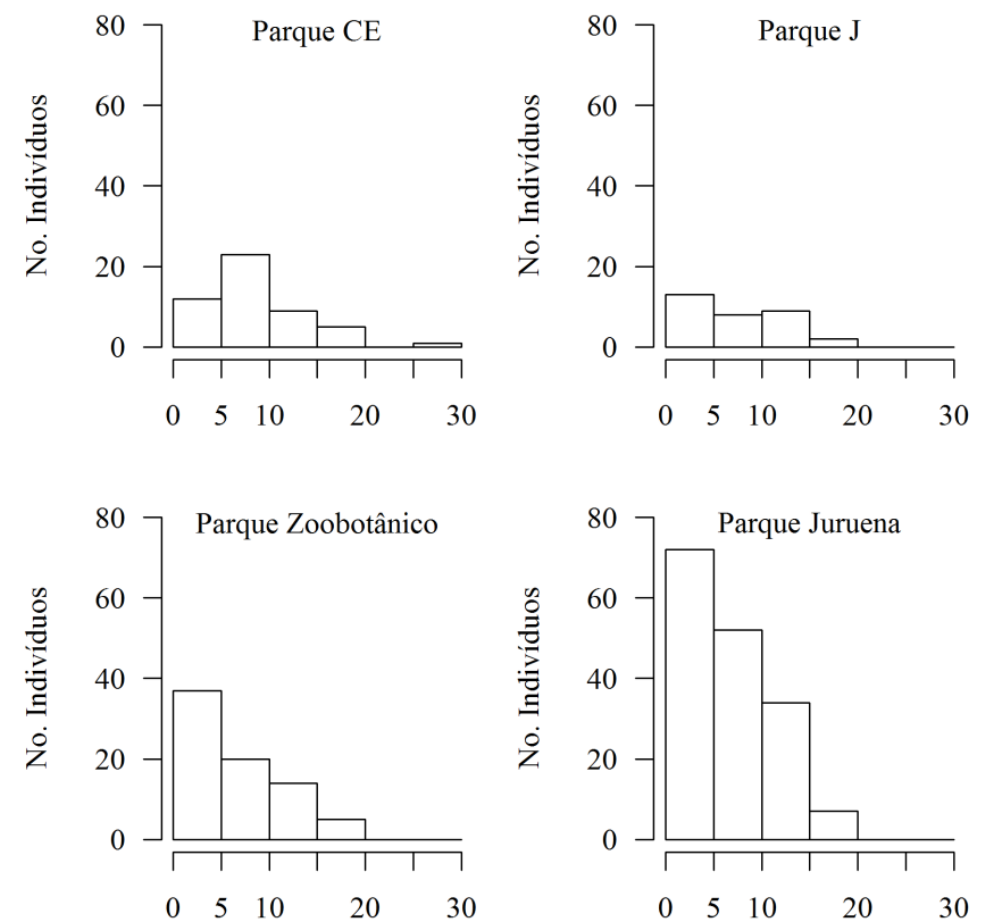

Diâmetro Altura do Peito $(\mathrm{cm})$

Diâmetro Altura do Peito $(\mathrm{cm})$

FIGURA 2: Distribuição por classes diamétricas dos indivíduos de Theobroma speciosum amostrados em três fragmentos florestais urbanos (na cidade de Alta Floresta) e no Parque Nacional do Juruena no norte do estado de Mato Grosso.

FIGURE 2: Distribution by diameter classes of individuals from Theobroma speciosum sampled in three urban forest fragments (in Alta Floresta city) and in the Juruena National Park in northern of Mato Grosso state.
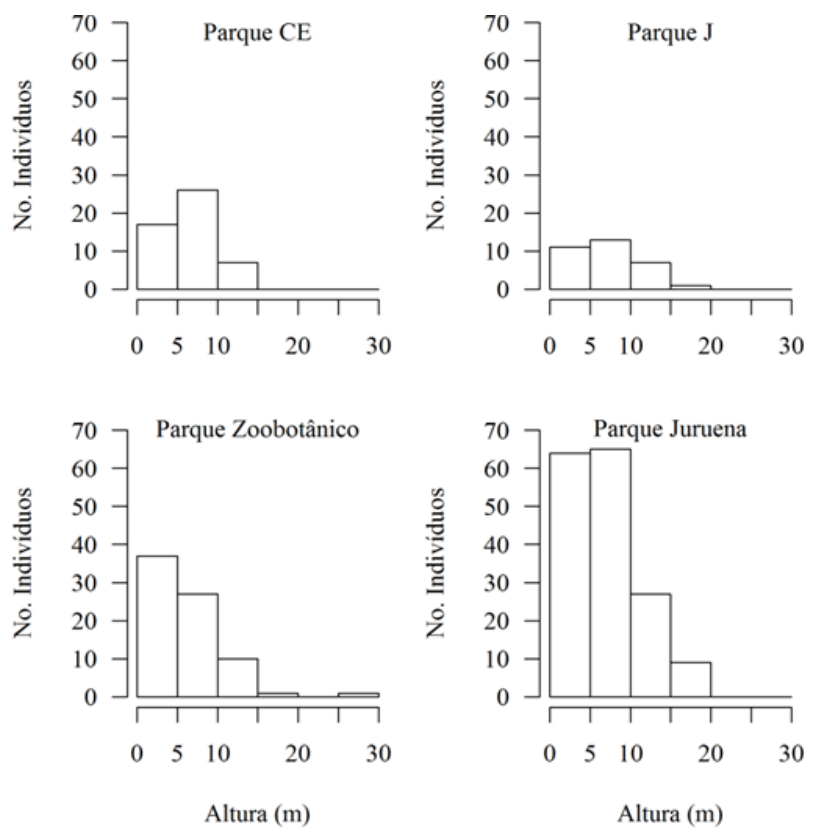

FIGURA 3: Distribuição por classes de altura dos indivíduos de Theobroma speciosum amostrados em três fragmentos florestais urbanos (na cidade de Alta Floresta) e no Parque Nacional do Juruena no norte do estado de Mato Grosso.

FIGURE 3: Distribution by height classes of individuals from Theobroma speciosum sampled in three urban forest fragments (in Alta Floresta city) and in the Juruena National Park in northern of Mato Grosso state. 
Esses dados permitem inferir que nos parques Zoobotânico e PNJu há uma maior taxa regenerativa, já que existem maiores proporções de indivíduos jovens, evidenciando uma possível mitigação dos efeitos da fragmentação, uma vez que a população encontra-se estável e em regeneração. Esses dois ambientes apresentam uma maior cobertura vegetal em comparação aos demais (Parques C/E e J). O PNJu consiste em floresta contínua e praticamente não há efeito de borda e no Parque Zoobotânico este efeito pode ser mitigado devido à sua maior área. Além disso, a floresta do PNJu não sofreu e não sofre perturbações antrópicas que causem o declínio dos indivíduos jovens.

Os indivíduos de Theobroma speciosum nos parques C/E e J não seguiram o padrão característico das espécies de florestas nativas ( $\mathrm{J}$ invertido), pois apresentaram um menor número de indivíduos distribuídos na primeira classe em relação às classes subsequentes. No parque urbano $\mathrm{C} / \mathrm{E}$, foram amostrados poucos indivíduos na menor classe de DAP $(0-5 \mathrm{~cm})$ e altura $(0-5 \mathrm{~m})$, ao contrário do parque J que apresenta um maior número de indivíduos na menor classe de DAP, porém, para as classes de altura o parque J assemelhase ao parque $\mathrm{C} / \mathrm{E}$ (Figuras 2 e 3). Tais resultados demonstram uma baixa taxa de regeneração da espécie nestes dois ambientes, evidenciando uma maior pressão antrópica em relação ao PNJu e ao fragmento do parque Zoobotânico.

Resultados semelhantes foram encontrados por Silva et al. (2013) com a mesma espécie e por Souza et al. (2011) com Theobroma subincanum. A baixa taxa de regeneração pode estar associada ao isolamento reprodutivo, visto que Theobroma speciosum é uma espécie alógama e apresenta barreiras fisícas que causam a autoincompatibilidade. Além disso, mudanças microclimáticas causadas pela fragmentação podem ocasionar dificuldades na germinação, no crescimento e na sobrevivência das plântulas. A fragmentação do habitat tem um efeito negativo muito maior sobre as espécies autoincompatíveis do que sobre as espécies autocompatíveis devido principalmente aos efeitos negativos sobre os agentes polinizadores (AGUILAR et al., 2006). Existem evidências do efeito negativo da fragmentação sobre a germinação (e.g., BRUNA, 2003) e sobre o recrutamento e sobrevivência das árvores menores (LAURANCE et al., 2004; NASCIMENTO et al., 2006).

TABELA 1: Comparação da estrutura de diâmetro e altura dos indivíduos de Theobroma speciosum entre quatro áreas florestais (três fragmentos florestais urbanos; Parques C/E, J e Zoobotânico - Zoo; e o Parque Nacional do Juruena - PNJu) no norte do estado de Mato Grosso.

TABLE 1: Comparisons of diameter and height structure of Theobroma speciosumbetween four forest areas (three urban forest fragments: C/E, J, Zoobotânico - Zoo Parks; and Juruena National Park - PNJu) in northern of Mato Grosso state. Differences between distributions were tested using Kolmogorov-Smirnov test (D).

\begin{tabular}{lcccc}
\hline Comparações & \multicolumn{2}{c}{ Distribuição Diamétrica } & \multicolumn{2}{c}{ Distribuição de Altura } \\
\cline { 2 - 5 } & $D$ & $P$ & $D$ & $P$ \\
\hline $\mathrm{PNJu} \times \mathrm{C} / \mathrm{E}$ & 0,20 & 0,08 & 0,15 & 0,39 \\
$\mathrm{PNJu} \times \mathrm{J}$ & 0,21 & 0,19 & 0,12 & 0,83 \\
$\mathrm{PNJu} \times$ Zoo & 0,12 & 0,41 & 0,13 & 0,31 \\
$\mathrm{C} / \mathrm{E} \times \mathrm{J}$ & 0,18 & 0,58 & 0,12 & 0,94 \\
$\mathrm{C} / \mathrm{E} \times$ Zoo & 0,26 & 0,03 & 0,16 & 0,42 \\
$\mathrm{~J} \times$ Zoo & 0,23 & 0,20 & 0,17 & 0,54 \\
\hline
\end{tabular}

Macacos e formigas predam os frutos de Theobroma speciosum antes da sua maturação (observação pessoal, T. L. VARELLA), prejudicando o banco de sementes que propiciaria a sucessão ecológica nesses ambientes. Lana et al. (2013) relatam que a perda de frutos e sementes pode gerar uma alteração na distribuição tipo $J$ invertido, indicando uma influência negativa no ingresso de indivíduos das menores classes para as de maiores. Este padrão em florestas nativas pode também ser influenciado por fatores bióticos, abióticos ou até mesmo fatores intrínsecos à espécie, dificultando a sua regeneração (SOUZA et al., 2011). 
A distribuição diamétrica dos indivíduos do parque C/E não diferiu significativamente do parque $\mathrm{J}$, através do teste de Kolmogorov-Smirnov $(D=0,18, P=0,58)$, entretanto, diferiu do parque Zoobotânico $(D=0,26, P=0,03)$. O PNJu tem uma distribuição parecida com a do parque $\mathrm{J}$ e do parque Zoobotânico, divergindo marginalmente $(D=0,2, P=0,08)$ do parque $\mathrm{C} / \mathrm{E}$. Para a distribuição de altura dos indivíduos, não houve diferença significativa entre os fragmentos em estudo, quando comparados par a par pelo teste de Kolmogorov-Smirnov conforme observado na Tabela 1.

O valor do Índice de Dispersão de Morisita $\left(I_{d}\right)$, para as quatro populações $(\mathrm{C} / \mathrm{E}, \mathrm{J}$, Zoobotânico e PNJu), indicou um padrão de distribuição espacial agregado $\left(I_{d}=3,23, I_{d}=5,35, I_{d}=10,07\right.$ e $I_{d}=1,69$, respectivamente), sugerindo que há, provavelmente, baixa taxa de competição intraespecífica na população, dispersão restrita de sementes próximas à planta-mãe, condições microclimáticas favoráveis ou fatores e distúrbios ambientais que limitam sua distribuição. Corroborando esses resultados, Silva et al. (2013) encontraram os mesmos padrões ao avaliarem a distribuição das espécies Theobroma speciosum e Theobroma subincanum no PNJu, o que pode ocorrer em função da heterogeneidade ambiental, pois, segundo Bernasol e Lima-Ribeiro (2010), as espécies se agrupam em lugares que oferecem as condições favoráveis para a sua reprodução e sobrevivência. Além disso, Bruzinga et al. (2013) relatam que o padrão de distribuição agregado pode indicar que a espécie apresenta uma dispersão limitada em relação à fonte de propágulos, ou é exigente em condições microambientais especificas.

O padrão de distribuição espacial agregada é comum entre outras espécies arbóreas (SOUZA; COIMBRA, 2005; ALMEIDA; CORTINES, 2008; BENASOL; LIMA-RIBEIRO, 2010; BRUZINGA et al., 2013). Dessa forma, os processos que atuam de forma negativa sobre a espécie estudada também podem ter efeitos pungentes sobre outras espécies com distribuição parecida.

Os indivíduos de menor porte de Theobroma speciosum, nas quatro populações estudadas, apresentaram agregações próximas aos indivíduos de maior porte (Figura 5). Esse agrupamento pode ser consequência do modo de dispersão dos frutos e sementes que são deixados pelos seus dispersores perto da planta-mãe. A distribuição espacial de sementes e plântulas depende da interação de fatores como a distribuição espacial das plantas-mãe, a existência de locais favoráveis à germinaçãoe e o padrão da chuva de sementes ao redor de cada planta-mãe (CRAWLEY, 1986; PARKER et al., 1997). Por outro lado, ambientes fragmentados ocasionam a extinção de espécies dispersoras, o que afeta a taxa de remoção das sementes, a distância de dispersão e o recrutamento das espécies de plântulas por esses animais, atingindo diretamente na distribuição da espécie nesses ambientes (AGUILAR et al., 2006; ANDREAZZI; PIRES; FERNANDEZ, 2009; RABELLO; RAMOS; HASUI, 2010).

Estudos que descrevem a forma de dispersão de Theobroma speciosum ainda não foram realizados, porém, foram observados durante as avaliações nos fragmentos urbanos que macacos consomem os frutos perto da planta-mãe ou até mesmo sem retirá-los das árvores. Também foram encontradas muitas plantas com seus frutos fixos no caule até o apodrecimento. Essas informações podem explicar o agrupamento de indivíduos menores próximos aos de maior porte, uma vez que dificilmente são observados frutos ou vestígios destes longe das árvores maduras da espécie. 

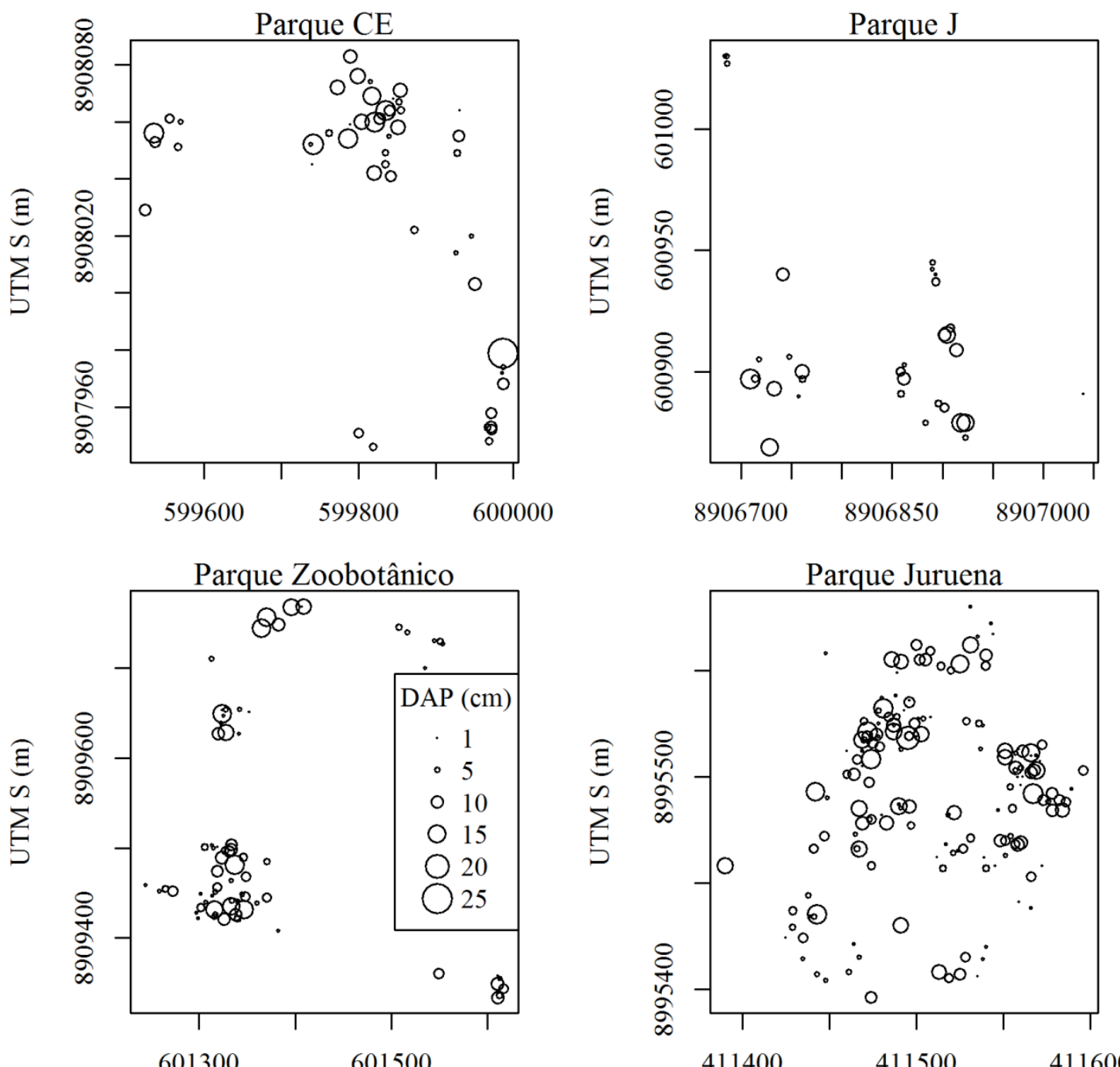

601300

601500

$$
411400
$$

411500

411600

\section{UTM W (m)}

FIGURA 5: Distribuição espacial dos indivíduos de Theobroma speciosum amostrados em três fragmentos florestais urbanos em Alta Floresta e no Parque Nacional do Juruena no norte do estado de Mato Grosso. O tamanho dos círculosrepresenta o diâmetro dos indivíduos.

FIGURE 5: Spatial distribution of individuals from Theobroma speciosum sampled in three urban forest fragments in Alta Floresta and in the Juruena National Park in northern of Mato Grosso state. Circlesize denotes thediameterofindividuals.

\section{CONCLUSÕES}

A distribuição diamétrica de Theobromaspeciosumfoi alterada em áreas fragmentadas (C/E e J) que sofreram efeito de borda ao passo que, em floresta não perturbada (Parque Nacional do Juruena) e fragmentos maiores (Parque Zoobotânico), a espécie manteve uma estrutura esperada com muitos indivíduos de tamanho pequeno. Quanto ao padrão de distribuição dos indivíduos, todas as populações apresentaram distribuição agregada, com os indivíduos jovens próximos aos prováveis parentais. Esses resultados indicam que a espécie é susceptível à fragmentação, provavelmente devido ao isolamento, pois apresenta autoincompatibilidade e uma dispersão zoocórica limitada. 


\section{AGRADECIMENTOS}

À Coordenação de Aperfeiçoamento de Pessoal de Nível Superior (CAPES) e à Fundação de Amparo à Pesquisa do Estado de Mato Grosso (FAPEMAT) pela concessão da bolsa de estudo a T. L.V. Ao BIONORTE - MT (Projeto Conhecimento, Uso Sustentável e Bioprospecção da Biodiversidade na Amazônia Meridional - Processo: 554330/2010-5) pelo apoio financeiro.

\section{REFERÊNCIAS}

AGUILAR, R. Plant reproductive susceptibility to habitat fragmentation: review and synthesis through a meta-analysis. Ecology Letters, Oxford, v. 9, n. 8, p. 968-980, 2006.

ALMEIDA, F. S.; CORTINES, E. Estrutura populacional e distribuição espacial de Piptadenia gonoacantha (Mart.) J. F. Mac br. Floresta e Ambiente, Seropédica, v. 15, n. 2, p. 18-23, 2008.

ANDREAZZI, C. S.; PIRES, A. S.; FERNANDEZ, A. S. Mamíferos e palmeiras neotropicais: interações em paisagens fragmentadas. Oecologia Brasiliensis, Rio de Janeiro, v. 13, n. 4, p. 554-574, 2009.

ARENAS, M. D. et al. Cobertura vegetal de Alta Floresta, Amazônia meridional matogrossense. Revista Espacios, Caracas, v. 36, n. 20, p. 15-26, 2015.

BERNASOL, W. P.; LIMA-RIBEIRO, M. S. Estrutura espacial e diamétrica de espécies arbóreas e seus condicionantes em um fragmento de cerrado sentido restrito no sudoeste goiano. Hoehnea, São Paulo, v. 37, n. 2, p. 181-198, 2010.

BRASIL. Ministério do Meio Ambiente. Secretaria de Políticas para o Desenvolvimento Sustentável. Programa de Zoneamento-Ecológico-Econômico. Caderno temático: biodiversidade no âmbito do zoneamentoecológico-econômico.Brasília: Ministério do Meio Ambiente, 2007. 240 p.

BROWER, J. E.; ZAR, J. H. Field and laboratory methods for general ecology. 2. ed. Dubique: Win. C. Brown, 1977. $226 \mathrm{p}$.

BRUNA, E. M. Are plant populations in fragmented habitats recruitment limited? Tests with an Amazonian herb. Ecology, Tempe,v. 84, n. 4, p. 932-947, 2003.

BRUZINGA, J. S. et al. Distribuição espacial de indivíduos adultos de pequi. ScientiaForestalis, Piracicaba, v. 41, n. 98, p. 249-256, 2013.

CAMPBELL, D. G. et al. Quantitative ecological inventory of terra firme and várzea tropical forest on the Rio Xingu, Brazilian Amazon. Brittonia, New York, v. 38, n. 4. p. 369-393, 1986.

CARVALHO, A. L.; FERREIRA, E. J. L.; LIMA, J. M. T. Comparações florísticas e estruturais entre comunidades de palmeiras em fragmentos de floresta primária e secundária da Área de Proteção Ambiental Raimundo Irineu Serra - Rio Branco, Acre, Brasil. Acta Amazônica, Rio Branco, v. 40, n. 4, p. 657-666, 2010.

CARVALHO, D. A. et al. Distribuição de espécies arbóreo-arbustivas ao longo de um gradiente de solos e topografia em um trecho de floresta ripária do Rio São Francisco em Três Marias, MG, Brasil. Revista Brasileira de Botânica, São Paulo, v. 28, n. 2, p. 329-345, 2005.

CARVALHO, F. A.; NASCIMENTO, M. T. Estrutura diamétrica da comunidade e das principais populações arbóreas de um remanescente de floresta atlântica sub montana (Silva Jardim-RJ, Bradil). Revista Árvore, Viçosa, MG, v. 33, n. 2, p. 327-337, 2009.

CAVAlCANTE, P. B. Frutas comestíveis da Amazônia. Belém: CEJUP; CNPq; Museu Paraense Emílio Goeldi, 1991. (Coleção Adolfo Ducke).

CRAWLEY, M. J. Plant ecology. Oxford: Blackwell, 1986.

DARDENGO, J. F. E. Diversidade genética, distribuição espacial e anatomia foliar de Theobroma L. em população natural no norte do estado de Mato Grosso. 2014. 88 f. Dissertação (Mestrado em Biodiversidade e Agroecossistemas Amazônicos) - Universidade do Estado de Mato Grosso, Alta Floresta, 2014.

DUCKE, A. As espécies brasileiras do gênero Theobroma L. Boletim TécnicoInstituto Agronômico, Campinas, v. 28, p. 1-20, 1953.

ESTOPA, R. A. et al. Diversidade genética em populações naturais de candeia (Eremanthus erythropappus 
(DC.) Macleish). Scientia Forestalis, Piracicaba, n. 70, p. 97-106, 2006.

FELFILI, J. M. Distribuição de diâmetros de quatro áreas de cerrado sensu stricto na Chapada do Espigão Mestre do São Francisco. In: FELFILI, J. M.; SILVA JÚNIOR, M. C. S. (Org.). Biogeografia do bioma cerrado: estudo fitofisionômico da Chapada do Espigão Mestre do São Francisco. Brasília: UnB, 2001. FERRÃO, J. E. M. Fruticultura tropical: espécies com frutos comestíveis. Lisboa: Instituto de InvestigaçãoCientífica Tropical, 2001. v. 3. 652p.

GILABERT-ESCRIVÁ, M. V. et al. Fatty acid and triacyl glycerol composition and thermal behavior of fats from seeds of Brazilian Amazonian Theobroma species. Journal of Science of Food and Agriculture, New Jersey, v. 82, n. 13, p. 1425-1431, 2002.

KREBS, C. J. Ecological methodology. New York: University of British Columbia, 1989. 653 p.

LANA, M. D. et al. Distribuição diamétrica de Escheweilera ovata em um fragmento de floresta ombrófila densa - Igarassu, PE. Revista Floresta, Curitiba, v. 43, n. 1, p. 59-68, 2013.

LAURANCE, W. F. et al. Pervasive alteration of tree communities in undisturbed Amazonian forests. Nature, London,v.428, p. 171-175, 2004.

LAURANCE, W. F.; VASCONCELOS, H. Consequências ecológicas da fragmentação florestal na Amazônia. Oecologia Brasiliensis, Rio de Janeiro, v. 13, n. 3, p. 434-451, 2009.

MACHADO, E. L. M. et al. Flutuações temporais nos padrões de distribuição diamétrica da comunidade arbóreo-arbustivo e de 15 populações em um fragmento florestal. Revista Árvore, Viçosa, MG, v. 34, n. 4, p. 723-732, 2010.

MIRANDA, I. P. A. et al. Levantamento quantitativo de espécies oleaginosas para produção de biodiesel na Reserva Extrativista do Capanã Grande - Município de Manicoré-AM. Relatório final do Projeto realizado na RESEX Capanã Grande no Município de Manicoré-AM. Manicoré: CTAgro; MCT; CNPq, 2004. 48 p.

MULLER-LANDAU, H. C. et al. Comparing tropical forest tree size distributions with the predictions of metabolic ecology and equilibrium models. Ecology Letters, Oxford, v. 9, n. 5, p. 589-602, 2006.

NASCIMENTO, H. E. M. et al. Effects of the surrounding matrix on tree recruitment in Amazonian Forest Fragments. Conservation Biology, Amsterdam, v. 20, n. 3, p. 853-860, 2006.

NASCIMENTO, H.; LAURANCE, W. F. Biomass dynamics in Amazonian Forest fragments. Ecological Applications, Tempe, v. 14, p. S127-S138, 2004.

NEPSTAD D. C. et al. Mortality of large trees and lianas following experimental drought in an amazon forest. Ecology, Brooklyn, v. 88, n. 9, p. 2259-2269, 2007.

OLIVEIRA, L. C. et al. Impactos da exploração seletiva de madeira em áreas em processo de fragmentação florestal na Amazônia Ocidental. Cerne, Lavras, v. 9, n. 2, p. 213-220, 2003.

PAGLIA, A. P.; FERNANDEZ, F. A. S.; MARCO, P. Efeitos da fragmentação de habitats: quantas espécies, quantas populações, quantos indivíduos, e serão eles suficientes? In: ROCHA, C. F. D. et al. (Org.). Biologia da conservação. São Carlos: RIMA, 2006. p. 281-316.

PARKER, K. C. et al. Population structure and spatial pattern of two coastal populations of Ocala sand pine (Pinusclausa). Journal of the Torrey Botanical Society, Lawrence, v. 124, n. 1, p. 22-33, 1997.

PARQUE NACIONAL DO JURUENA. [website]. Disponível em: $<$ http://www.parquenacionaldojuruena. com.br>. Acessoem: 20 jun. 2016.

R DEVELOPMENT CORE TEAM. R: a language and environment for statistical computing. Vienna: R Foundation for Statistical Computing, 2005.

RABELLO, A.; RAMOS, F. N.; HASUI, E. Efeito do tamanho do fragmento na dispersão de sementes de copaíba (Copaifera langsdorffii Delf.). Biota Neotropica, Campinas, v. 10, n. 1, p. 47-54, 2010.

RIBEIRO, J. F.; FONSECA, C. E. L.; SILVA, J. C. S. Cerrado: caracterização e recuperação de matas de galeria. Planaltina: Embrapa Cerrados, 2001.

SADDI, N. A primeira contribuição sobre a flora de Humboldt (Aripuanã, Mato Grosso). In: CONGRESSO NACIONAL DE BOTÂNICA, 26., 1975, Rio de Janeiro. Trabalhos... Rio de Janeiro: Academia Brasileira de Ciências, 1977.

SILVA, B. M. et al. Estrutura e padrões de distribuição espacial de duas espécies de Theobroma em um parque de preservação permanente no norte do estado de Mato Grosso. Enciclopédia Biosfera, Goiânia, v. 9, n. 17, p. 2789, 2013. 
SILVA JÚNIOR, M. C. Fitossociologia e estrutura diamétrica da mata de galeria do Taquara, na reserva ecológica do IBGE, DF. Revista Árvore, Viçosa, MG, v. 28, n. 3, p. 419-428, 2004.

SOUZA, C. S. C. et al. Caracterização estrutural da espécie Theobroma subincanum Mart (Cupuí) na reserva extrativista do Rio Cajari, Amapá-Brasil. In: SIMPÓSIO LATINO-AMERICANO SOBRE MANEJO FLORESTAL, 5., 2011, Santa Maria. Anais... Santa Maria: UFSM, 2011. p. 799-806.

SOUZA, J. P.; COIMBRA, F. G. Estrutura populacional e Distribuição espacial de Qualea parviflora Mart. em um Cerrado sensu stricto. Biosciência, Taubaté, v. 21, n. 2, p. 65-70, 2005.

TARIFA, J. R. Mato Grosso: clima - análise e representação cartográfica.Cuiabá: Entrelinhas, 2011. 102 p. TOLEDO, J. J. et al. Tree mode of death in Central Amazonia: effects of soil and topography on tree mortality associated with storm disturbances. Forest Ecology and Management, Amsterdam, v. 263, p. 253-261, 2012.

VASCONCELOS, H. L. et al. Long-term effects of forest fragmentation on Amazonian ant communities. Journal of Biogeography, Oxford,v. 33, n. 8, p. 1348-1356, 2006. 